TRANSACTIONS OF THE

AMERICAN MATHEMATICAL SOCIETY

Volume 202, 1975

\title{
$H$-CLOSED EXTENSIONS. II $\left({ }^{1}\right)$
}

BY

\section{JACK R. PORTER AND CHARLES VOTAW}

\begin{abstract}
The internal structure and external properties (in terms of other $H$-closed extensions) of the Fomin extension $\sigma X$ of a Hausdorff space $X$ are investigated. The relationship between $\sigma X$ and the Stone-Čech compactification of the absolute of $X$ is developed and used to prove that a $\sigma X$-closed subset of $\sigma X \backslash X$ is compact and to show the existence of a Tychonoff space $Y$ such that $\sigma X \backslash X$ is homeomorphic to $\beta Y Y$. The sequential closure of $X$ in $\sigma X$ is shown to be $X$.

It is known that $\sigma X$ is not necessarily projectively larger than any other strict $H$-closed extension of $X$; a necessary and sufficient condition is developed to determine when a $H$-closed extension of $X$ is projectively smaller then $\sigma X$. A theorem by Magill is extended by showing that the sets of $\theta$-isomorphism classes of $H$-closed extensions of locally $H$-closed spaces $X$ and $Z$ are lattice isomorphic if and only if $\sigma X \backslash X$ and $\sigma Z \backslash Z$ are homeomorphic. Harris has characterized those simple Hausdorff extensions of $\boldsymbol{X}$ which are subextensions of the Katětov extension. Characterizations of Hausdorff (not necessarily simple) extensions of $X$ which are subextensions of $\boldsymbol{H}$-closed extensions $\theta$-isomorphic and $S$-equivalent to the Katětov extension are presented.
\end{abstract}

In this paper, we investigate properties of the Fomin extension and the complete upper semilattice of $H$-closed extensions of a Hausdorff space; some of our results are obtained by use of the absolute of a Hausdorff space. For a Hausdorff space $X$, the sequential closure of $X$ in the Fomin extension $\sigma X$ is shown to be $X$ and subsets of $\sigma X \backslash X$ which are closed in $\sigma X$ are shown to be compact. If $Y$ is an $H$-closed extension, it is known ([IF], [L], [PT]) that the identity function on $X$ can be extended to a continuous function from the Katětov extension $\kappa X$ to $Y$ whereas the corresponding result with $\kappa X$ replaced by $\sigma X$ is false even if $Y$ is a strict extension. A necessary and sufficient condition is given for the identity function on $X$ to extend to a continuous function from $\sigma X$ to $Y$.

Received by the editors May 31, 1973 and, in revised form, February 14, 1974.

AMS (MOS) subject classifications (1970). Primary 54D25, 54D40; Secondary 54C20, 54D35, 54D55, 54G05.

Key words and phrases. $H$-closed extension, Katětov extension, Fomin extension, absolute, projective cover.

(1) The research of the first author was partially supported by a University of Kansas research grant and of the second author by a National Science Foundation Traineeship. 
We prove a result similar to a theorem by Magill [M] by showing the sets of $\theta$-isomorphism classes of $H$-closed extensions of locally $H$-closed spaces $X$ and $Y$ are lattice isomorphic if and only if $\sigma X \backslash X$ and $\sigma Y \backslash Y$ are homeomorphic. In addition, we prove that for a Hausdorff space $X$, there is a Tychonoff space $Y$ with the property that the sets of $\theta$-isomorphism classes of $H$-closed extensions of $X$ and $Y$ are lattice isomorphic. Given a simple Hausdorff extension $T$ of a space $X$, Harris [H] has found necessary and sufficient conditions for $T$ to be isomorphic, as extensions of $X$, to a subspace of the Katětov extension; we approach this setting from a different direction by characterizing, in terms of $H$-closed extensions, those Hausdorff extensions of $X$ satisfying each of these conditions.

This paper is a continuation of [PV], and the definitions and notation developed in [PV] will be used in this paper. Also, the numbering in this paper is a continuation of the numbering in [PV]. In particular, $X$ will always denote a Hausdorff space. There are a few additional definitions and facts that will be helpful in this paper.

Banaschewski [B] has shown for a semiregular Hausdorff space $X$ that $(\kappa X)_{s}$ is a minimal Hausdorff extension of $X$, denoted by $\mu X$. Flachsmeyer ([F1], [F2]) has shown that $\mu X$ is a special extension $\sigma_{B} X$ of Fomin-Shanin ([Fo], [S1], [S2]) type with respect to the base $B$ of all regular open sets. Correspondingly, $\mu X$ is called the Banaschewski-Fomin-Shanin extension; by 1.6, $\mu X=(\kappa X)_{s}=(\sigma X)_{s}$.

(1.8) If $Y$ is a Hausdorff extension of $X$ and $f: Y \rightarrow Z$ is a continuous function such that $f \backslash X$ is a homeomorphism onto $f(X)$, then $f(Y \backslash) \subseteq Z \backslash f(X)$.

(1.9) Let $F$ be an open filter on $X$ and $G=\bigcap\{U: U$ is an open ultrafilter on $X$ and $F \subseteq U$ \}. Then

$$
G=\{U: U \text { open in } X \text { and } \operatorname{int}(\operatorname{cl}(U)) \in F\}=F \vee D
$$

where $D=\{U: U$ open in $X$ and $X=\operatorname{cl}(U)\}$.

Proof. Since $D$ is contained in every open ultrafilter, then $F \vee D \subseteq G$. Let $U$ be open in $X$ such that $\operatorname{int}(\operatorname{cl}(U)) \notin F$. Then $(X \backslash \operatorname{cl}(U)) \cap F \neq \varnothing$ for all $F \in F$. For some open ultrafilter $U, U \supseteq F \cup\{X \backslash \mathrm{cl}(U)\}$. This shows $G \subseteq\{U: U$ open in $X$ and int(cl(U)) $\in F\}$. Now, let $U$ be open in $X$ such that $\operatorname{int}(\operatorname{cl}(U)) \in F$. Then $X \backslash b d U \in D$ and $\operatorname{int}(\operatorname{cl}(U)) \cap(X \backslash b d U)=U \in F V$ $D$. Hence, $\{U: U$ open in $X$ and $\operatorname{int}(\operatorname{cl}(U)) \in F\} \subseteq F \vee D$. This completes the proof.

The next fact is an easy, consequence of (1.9).

(1.10) If $U$ is an open ultrafilter on $X$ and $V$ is an open set such that $\operatorname{int}(\operatorname{cl}(V)) \in U$, then $V \in U$.

The authors wish to thank the referee for his useful suggestions. 
5. Internal properties of $\odot X$. Let $X$ be a Hausdorff space. $\theta X$ is used to denote $\{q: q$ is an open ultrafilter on $X\}$. For each open set $U$ in $X$, let $G_{U}=\{q \in \theta X: U \in q\}$. $\left\{G_{U}: U\right.$ open in $\left.X\right\}$ forms a base for an extremally disconnected, compact Hausdorff topology on $\theta X$ [IF, Theorems 1,2]. The subspace $\{q \in \theta X: q$ is fixed $\}$ is denoted as $p X$ and called the absolute of $X$; $p X$ is dense in $\theta X$ [IF, Theorem 4]. By Exercise $6 \mathrm{M}$ in [GJ], $\theta X=\beta p X$ as extensions of $p X$ and $p X$ is an extremally disconnected, Tychonoff space. Define a function $\pi$ from $\theta X$ onto $\sigma X$ by $\pi(q)=q$ if $q$ is free and $\pi(q)=$ $x$ if $N_{x} \subseteq q$; this extends the function $\pi$, defined in [IF] with domain $p X$ and range $X$.

Recall that a function $f: Y \rightarrow Z$ is irreducible if $f$ is onto and no proper closed subset of $Y$ is mapped onto $Z$, is compact if $f^{-1}(z)$ is compact for each $z \in Z$, and is perfect if $f$ is closed and compact.

Theorem 5.1 [IF, TheOREM 10]. $\pi \mid p X: p X \rightarrow X$ is a $\theta$-continuous, irreducible, perfect function.

By a proof similar to the proof of Theorem 10 of [IF], these properties of $\pi \mid p X$ can be extended to $\pi$.

TheOREM 5.2. $\pi: \theta X \rightarrow \sigma X$ is a $\theta$-continuous, irreducible, perfect function.

It is clear that $\pi$ is a bijection when the domain is restricted to the remainder $\theta X \backslash p X$ and the range to the remainder $\sigma X \backslash X$. In fact, more is true, as indicated in the next lemma (the proof is straightforward).

LEMMA 5.3. (a) For each open set $U, \pi\left(G_{U}\right) U X=O_{U} \backslash U$ and $\pi^{-1}\left(O_{U} \backslash U\right)=G_{U} \backslash p X$.

(b) $\pi \mid(\theta X \backslash p X): \theta X \backslash p X \rightarrow \sigma X \backslash X$ is a homeomorphism.

THEOREM 5.4. Suppose there is a continuous function from $\sigma X$ onto an H-closed extension $Y$ of $X$ that leaves $X$ pointwise fixed. Let $F \subseteq Y X$. The following are equivalent:

(a) $F$ is closed in $Y$.

(b) $F$ is compact.

(c) $\bigcap\left\{O^{y}: y \in F\right\}$ is a free open filter, and if $\bigcap_{\left\{O^{y}: y \in F\right\} \text { meets } O^{z}}$ for some $z \in M X$, then $z \in F$.

Proof. Let $f: \sigma X \rightarrow Y$ be the continuous function that leaves $X$ pointwise fixed and $F=\bigcap\left\{O^{y}: y \in F\right\}$.

(a) implies (b). By $5.3(\mathrm{~b})$, it suffices to show that $\pi^{-1}\left(f^{-1}(F)\right)$ is closed in $\theta X$ since $\theta X$ is compact. By 5.3(b), $\pi^{-1}\left(f^{-1}(F)\right)$ is closed in $\theta X \mid p X$. Let $q \in p X$ and $N_{x} \subseteq q$ where $x \in X$. Now $O_{V} \cap f^{-1}(F)=\varnothing$ for some 
$V \in N_{x}$, implying $G_{V} \cap \pi^{-1}\left(f^{-1}(F)\right)=\varnothing$. Thus, $\pi^{-1}\left(f^{-1}(F)\right)$ is closed in $\theta X$. (b) implies (c). Clearly $F$ is an open filter on $X$. Let $x \in X$. There are disjoint open sets $R$ and $S$ in $Y$ such that $x \in R$ and $F \subseteq S$. Now $x \in R \cap X, S \cap X \in F$, and $(R \cap X) \cap(S \cap X)=\varnothing$; so, $F$ is free. Suppose $z \in Y \backslash X$ and $z \notin F$. There are disjoint open sets $R$ and $S$ in $Y$ such that $z \in R$ and $F \subseteq S$. Now $R \cap X \in O^{z}$ and $S \cap X \in F$, implying $F$ does not meet $O^{z}$.

(c) implies (a). Since $F$ is a free open filter, the closure of $F$ in $Y$ is contained in $Y \backslash X$. Let $z \in Y(X \cup F)$. Then $O^{z}$ does not meet $F$; so, there are disjoint open sets $V \in O^{z}$ and $V \in F$. Now $o(V) \cap o(V)=\varnothing, z \in o(V)$, and $F \subseteq o(V)$. So, $z \notin \mathrm{cl}_{y} F$. Hence, $F$ is closed in $Y$.

The authors are indebted to the referee for a suggestion that improved Theorem 5.4. By Theorem 4 in [F1], the $Y$ in Theorem 5.4 can be replaced by $\sigma_{B} X$ where $B$ is a $\pi$-basis. The $H$-closed extensions $Y$ of $X$ that satisfy the hypothesis of Theorem 5.4 are characterized in Theorem 6.2. In the case that the $H$-closed extension $Y$ of $X$ is $\sigma X$ and $F \subseteq \sigma X \backslash X$, the first part of 5.4(c) is equivalent to $F$ being free; using the fact that an open filter $F$ meets an open ultrafilter $U$ if and only if $F \subseteq U$, the second part of 5.4(c) is equivalent to $F$ being saturated (see the paragraph before Remark 2.2 ). Thus, we have the following extension of Theorem 2.1.

COROLLARY 5.5. Let $R$ be an equivalence relation on $\sigma X$ such that $R(x)=\{x\}$ for $x \in X$. There is an H-closed extension $Y$ of $X$ such that $R=$ $R_{Y}$ if and only if $R(y)$ is compact for each $y \in \sigma X$.

A Hausdorff space $Y$ is locally $H$-closed [0] if every point has an $H$-closed neighborhood. Porter [Po] showed that a Hausdorff space $Y$ is locally $H$-closed if and only if $Y$ is an open subspace in each of its $H$-closed extensions. Other characterizations of locally $\mathrm{H}$-closed spaces are provided in the next theorem.

THEOREM 5.6. The following are equivalent for a Hausdorff space $X$.

(a) $X$ is locally H-closed.

(b) $X$ is open in $\sigma X$.

(c) $\sigma X \backslash X$ is compact.

(d) $p X$ is locally compact.

Proof. That (a) and (b) are equivalent $\mid$ follows from the proof of Theorem 3.1 of [Po] , and that (b) and (c) are equivalent follows from Theorem 5.4. If $p X$ is locally compact, then $\theta X \backslash p X$ is compact, and by Theorem 5.2, $\pi(\theta X \backslash p X)=\sigma X \backslash X$ is compact. This shows (d) implies (b). By the proof of Theorem 5.4 ((a) implies (b) part), if $\sigma X \backslash X$ is closed in $\sigma X$, then $\pi^{-1}(\sigma X \backslash X)$ $=\theta X \backslash p X$ is closed in $\theta X$; this shows that $p X$ is locally compact and that (b) implies (d). 
Corollary 5.7. A Hausdorff space $X$ is almost H-closed (i.e., card $\sigma X \backslash X \leqslant 1$ [Po]) if and only if $p X$ is almost compact.

Here is how another property translates between $X$ and $p X$.

THEOREM 5.8. For a Hausdorff space $X$, a point $q \in p X$ is isolated if and only if $\pi(q)$ is isolated in $X$.

PRoof. The proof is straightforward.

The referee has asked the question of whether $p(\sigma X)=\sigma(p X)$ for an arbitrary Hausdorff space $X$. The question was motivated by the fact that $p(\sigma X)=\beta(p X)$; so, the question is reduced to whether $\sigma(p X)=\beta(p X)$ for an arbitrary Hausdorff space $X$. Here is a solution to the question.

THEOREM 5.9. Let $X$ be a Hausdorff space. $p(\sigma X)=\sigma(p X)$ if and only if the set of nonisolated points of $p X$ is compact.

Proof. Since $p(\sigma X)=\sigma(p X)$ if and only if $\sigma(p X)=\beta(p X)$, then, by Theorem 11 in [K2], $p(\sigma X)=\sigma(p X)$ if and only if the set of nonisolated points of $p X$ is compact.

Thus, when $X$ is a Hausdorff space that is not $H$-closed and has no isolated points (e.g., the real numbers with the usual topology), $p(\sigma X) \neq \sigma(p X)$. On the other hand, if $X$ is a semiregular Hausdorff space, then by Corollary 9 in [IF], $p(\mu X)=\beta(p X)$. But every regular nowhere dense closed set in $p X$ is compact; so, by Theorem $6.4 \mathrm{in}[\mathrm{PT}], \beta(p X)=\mu(p X)$. Thus, for every semiregular Hausdorff space $X, p$ and $\mu$ commute, i.e., $\mu(p X)=p(\mu X)$.

It is well known [GJ, Example 9K6] that each Tychonoff space $S$ is homeomorphic to $\beta T \backslash T$ for some Tychonoff space $T$. It seems natural to inquire whether each Hausdorff space $X$ is homeomorphic to $\sigma Y \backslash Y$ or $k Y \backslash Y$ for some Hausdorff space $Y$. This is false for $k Y$, as $\kappa Y Y$ is discrete; the next theorem shows it is also false for $\sigma Y$.

THEOREM 5.10. (a) For a Hausdorff space $X, \sigma X \backslash X=\beta(p X) \backslash p X$ for the Tychonoff, extremally disconnected space $p X$.

(b) If $Y$ is a Tychonoff, extremally disconnected space, then $\beta Y \backslash Y=$ $\sigma Y \backslash Y$.

Proof. The proof of (a) follows from Lemma 5.3.

The proof of (b) follows from Lemma 5.3 and the fact [IF, Corollary 2] that for a Tychonoff, extremally disconnected space $Y, \pi p Y: p Y \rightarrow Y$ is a homeomorphism.

COROllary 5.11. For a Hausdorff space $X, \sigma X \backslash X=\sigma(p X) \backslash p X$.

It follows by Theorem 5.10 that the class, henceforth denoted as $\Sigma$, of 
spaces $\sigma X \backslash X$ as $X$ ranges over all Hausdorff spaces is the same as the class of spaces $\beta Y \backslash Y$ where $Y$ ranges over all Tychonoff, extremally disconnected spaces. Since $\beta p X$ is extremally disconnected, $\Sigma$ is a subclass of the class of Tychonoff, zero-dimensional spaces. It is a proper subclass since spaces in $\Sigma$ possess the property that a convergent sequence is eventually constant [Wi, 15G3]; in particular, the space of rational numbers with the usual topology is not an element of $\Sigma$.

PROBLEM. Find an internal characterization of spaces which are the remainders of Stone-Čech compactifications of extremally disconnected spaces.

The sequential closure of a normal Hausdorff space $Y$ in $\beta Y$ is $Y$ [W, Theorem 8.3.2]; the corresponding fact for Tychonoff spaces is not necessarily true (an example is the deleted Tychonoff plank). However, if $Y$ is a Tychonoff space, then $\beta Y$ is not first countable at any point of $\beta Y \backslash Y$. The analogous results for $\sigma X$ are contained in the next theorem and corollary.

Theorem 5.12. The sequential closure of the Hausdorff space $X$ in $\sigma X$ and in $\mathrm{k} X$ is $X$.

Proof. Assume that $\left(x_{n}\right)$ is a sequence in $X$ converging to some point $p$ in $\sigma X \backslash X$. Using induction, it is straightforward to derive a subsequence $\left(z_{n}\right)$ of $\left(x_{n}\right)$ and a decreasing family $\left\{U_{n}: n \in N\right\}$ of open sets of $p$ with the properties $\left\{z_{1}, \cdots, z_{n}\right\} \cap \mathrm{cl}_{X}\left(U_{n+1}\right)=\varnothing$ and $z_{n} \in U_{n}$ for all $n \in \mathrm{N}$. Let $A=\left\{z_{n}: n \in \mathrm{N}\right\} ; A$ is closed in $X$ since $A \cup\{p\}$ is closed in $\sigma X$. Either $X \backslash A$ or $\operatorname{int}_{X} A$ belongs to $p$. But $X \backslash A$ contains no member of the converging subsequence $\left(z_{n}\right)$; hence, int $_{X} A \in p$. Since, for all $n \in \mathrm{N}$, $\left(\right.$ int $\left._{X} A\right)$ $\cap U_{n} \neq \varnothing$ and (int $\left.X_{X} A\right) \cap\left(U_{n} \mid c_{X}\left(U_{n+1}\right)\right) \subseteq\left\{z_{n}\right\}$, there is a subsequence $\left(y_{n}\right)$ of $\left(z_{n}\right)$ converging to $p$ with the property that $\left\{y_{n}\right\}$ is open in $X$ for all $n$. The sets $\left\{y_{2 n}: n \in \mathbf{N}\right\}$ and $\left\{y_{2 n+1}: n \in \mathbf{N}\right\}$ are disjoint, open sets in $X$, and since both sets, as sequences, converge to $p$, both belong to $p$, which is a contradiction. The proof for $k X$ is similar.

Corollary 5.13. Let $X$ be a Hausdorff space. $\sigma X$ (resp., $\kappa X$ ) is not first countable at any point of $\sigma X \backslash X$ (resp., $\kappa X \backslash X)$ ).

6. Regularity conditions and $\sigma X$. Let $Y$ be an $H$-closed extension of $X$ and $f: \kappa X \rightarrow Y$ the Katětov function of $Y$. The Fomin extension $\sigma X$ of $X$ is simply the underlying set of $\kappa X$ with a coarser topology; hence, the question arises as to when the Katětov function is continuous on $\sigma X$. In this section, we derive an answer. The answer is related to a modified regularity condition. (Note that if $g: \sigma X \rightarrow Y$ is a continuous function extending the identity on $X$, then $g$ is the Katětov function of $Y$.)

We now define a regularity condition related to the continuity of the 
Katětov function on $\sigma X$. A space $Z$ is regular relative to a subset $A$ if for each $z \in Z$ and each open set $U$ of $z$, there is an open set $V$ such that

$$
z \in V \cup\left(A \cap \operatorname{int}_{z} \mathrm{cl}_{z} V\right) \subseteq V \cup\left(A \cap \mathrm{cl}_{z} V\right) \subseteq U .
$$

If $Z$ is regular relative to $Z$, then $Z$ is regular. This definition is related to $\sigma X$ in the next theorem, but first we need a lemma.

LEMma 6.1. Let $f: \kappa X \rightarrow Y$ be the Katětov function of an H-closed extension $Y$ of $X$. Then for each open set $U$ in $X, o_{Y} U \subseteq f\left(O_{U}\right)=U U$ $\left(\mathrm{cl}_{Y} U \mathrm{UX}\right)$.

Proof. Let $y \in o_{Y} U$. Then $O^{y} \subseteq z$ for some $z \in \kappa X$; hence $U \in z$ and $z \in O_{U}$. Thus, $y=f(z)$ and $o_{Y} U \subseteq f\left(O_{U}\right)$. Now, let $z \in O_{U}$ and $y=$ $f(z)$. Then $O^{y} \subseteq z$, so $U \cap V \neq \varnothing$ for every $V \in O^{y}$, implying $y \in \operatorname{cl}_{Y} U$. It follows by 1.8 that $f\left(O_{U}\right) \subseteq U \cup\left(\mathrm{cl}_{Y} U \backslash X\right)$. Conversely, it suffices to show $\operatorname{cl}_{Y} U X \subseteq f\left(O_{U}\right)$ since $U \subseteq o_{Y} U$. So, let $y \in \operatorname{cl}_{Y} U X$. Then $O^{y}$ is a free open filter on $X$ and $U \cap V \neq \varnothing$ for every $V \in O^{y}$. Thus, $O^{y} \cup\{U\} \subseteq z$ for some free open ultrafilter $z$ on $X$. Now $z \in O_{U}$ and $f(z)=y$, so $\mathrm{cl}_{Y} U X \subseteq f\left(O_{U}\right)$.

THEOREM 6.2. Let $f: \kappa X \rightarrow Y$ be the Katětov function of an H-closed extension $Y$ of $X$. $f$ factors through $\sigma X$ (i.e., $f: \sigma X \rightarrow Y$ is continuous) if and only if $Y$ is regular relative to $Y X$.

Proof. Suppose $f$ factors through $\sigma X$. Let $y \in Y$ and $U$ an open set containing $y$. Then, by Theorems 2.1 and $5.4, f^{-1}(y)$ is a compact subset of $\sigma X$. It follows that there is an open set $V$ in $X$ such that $f^{-1}(y) \subseteq$ $O_{V}$ and $f\left(O_{V}\right) \subseteq U$. If $y \in X$, then $y \in V \subseteq o_{Y} V$. If $y \notin X$, then $V \in$ $\cap f^{-1}(y)$; since $f^{-1}(y)=\left\{z: z\right.$ is open ultrafilter and $\left.O^{y} \subseteq z\right\}$, then, by 1.9, int ${ }_{X} \mathrm{cl}_{X} V \in O^{y}$. Thus, by 1.1 ,

$$
y \in\left(\text { int }_{Y} \mathrm{cl}_{Y} o_{Y} V\right) \backslash X=o_{Y}\left(\text { int }_{X} \mathrm{cl}_{X} V\right) \backslash X .
$$

Since $f\left(O_{V}\right) \subseteq U$, then by 1.1 and Lemma 6.1, $\left(o_{Y} V\right) \cup\left(\mathrm{cl}_{Y} o_{Y} V\right) \backslash X \subseteq U$. Thus, $Y$ is regular relative to $Y X$. Conversely, suppose $Y$ is regular relative to $Y X X$ and that $f(z)=y$. Let $U$ be an open set containing $y$. There is an open set $V$ such that

$$
y \in V \cup\left(\text { int }_{Y} \mathrm{cl}_{Y} V X\right) \subseteq V \cup\left(\mathrm{cl}_{Y} V X\right) \subseteq U .
$$

Let $W=V \cap X$. If $y \in X$, then $z \in W \subseteq O_{W}$. Suppose $y \notin X$. By 1.1, int $_{Y} \mathrm{cl}_{Y} V=o_{Y}\left(\right.$ int $\left._{X} \mathrm{cl}_{X} W\right)$. Since $O^{y} \subseteq z$, then int $_{X} \mathrm{cl}_{X} W \in z$. By 1.10, $W \in z$ implying $z \in O_{W}$. By Lemma 6.1, $f\left(O_{W}\right)=W \cup\left(\mathrm{cl}_{Y} W X\right) \subseteq V \cup$ $\left(\operatorname{cl}_{Y} \backslash X\right) \subseteq U$. This shows that $f$ factors through $\sigma X$. 
Now $\sigma X$ is regular relative to $\sigma X \backslash X$ (it will be noted below that $\sigma X$ possesses an even stronger property), so it is clear from the preceding theorem that $\sigma X$ is a projective maximum among the $H$-closed extensions $Y$ of $X$ which are regular relative to $Y X$.

Also, the concept of regular relative to a subset is related to the question of whether the Katětov function $f$ of a minimal Hausdorff extension $Y$. of a semiregular Hausdorff space $X$ can be factored through the BanaschewskiFomin-Shanin extension $\mu X$, i.e., $(\sigma X)_{s}$. Again, note that if $g: \mu X \rightarrow Y$ is a continuous function extending the identity map on $X$, then $g$ is the Katetov function of. $Y$.

THEOREM 6.3. Let $X$ be a semiregular Hausdorff space and $Y$ a minimal Hausdorff extension of $X$. The Katětov function $f$ of $Y$ factors through $\mu X$ if and only if $Y$ is regular relative to $Y X$.

PRoof. If $f$ factors through $\mu X$, then $f$ factors through $\sigma X$ implying $Y$ is regular relative to $Y \backslash X$ by Theorem 6.2. Conversely, suppose $Y$ is regular relative to $Y X$. By Theorem 6.2, $f$ factors through $\sigma X$. Suppose $z \in$ $\sigma X, f(z)=y$ and $U$ is a regular-open set in $Y$ containing $y$. For some $V \in$ $z, y=f(z) \in f\left(O_{V}\right) \subseteq U$. By 1.1, int ${ }_{\sigma X} \mathrm{cl}_{\sigma X} O_{V}=O_{W}$ where $W=$ int $_{X} \mathrm{cl}_{X} V$. So, $z \in O_{W}$ and $y \in f\left(O_{W}\right)=W \cup \mathrm{cl}_{Y} W X$. Since $O_{W}=W \cup O_{V}$ by 1.2, then it follows that $f\left(O_{W}\right) \subseteq \operatorname{int}_{Y} \operatorname{cl}_{Y} U=U$. Thus, $f$ factors through $\mu X$.

Recall [BPS] that a regular filter $F$ on $X$ is an open filter with the property that for each $U \in F$, there is a $V \in F$ such that $\operatorname{cl}_{X} V \subseteq U$.

THEOREM 6.4. Let $Y$ be an extension of $X$. Then $Y$ is regular if and only if $Y$ is regular relative to $Y X$ and $O^{y}$ is a regular filter on $X$ for each $y \in Y$.

Proof. It is easy to verify that a regular space $Y$ satisfies the two conditions. To prove the converse, let $y \in U \subseteq Y$ where $U$ is open in $Y$. Since $U \cap X \in O^{y}$, then there is an open set $W$ in $Y$ that contains $y$ and $\operatorname{cl}_{X}(W \cap X) \subseteq U \cap X$ and $W \subseteq U$. There is an open set $V$ in $Y$ such that

$$
y \in V \cup\left(\text { int }_{Y} \mathrm{cl}_{Y} V\right) \cup\left(X \subseteq V \cup\left(\mathrm{cl}_{Y} V\right) \backslash X \subseteq W .\right.
$$

Now, $y \in \operatorname{int}_{Y} \mathrm{cl}_{Y} V \subseteq \operatorname{cl}_{Y} V=\left(\left(\operatorname{cl}_{Y} V\right) \cap X\right) \cup\left(\operatorname{cl}_{Y} V X\right)$. Since

$$
\left(\mathrm{cl}_{Y} V\right) \cap X=\left(\mathrm{cl}_{Y}(V \cap X)\right) \cap X=\operatorname{cl}_{X}(V \cap X) \subseteq \mathrm{cl}_{X}(W \cap X) \subseteq U \cap X,
$$

then it follows that $\operatorname{cl}_{Y} V \subseteq U$. Thus, $X$ is regular.

If $X$ is a Tychonoff space, then by Theorem 6.2, $\sigma X$ is projectively larger than any compactification of $X$, including the Stone-Čech compactification. So, $\sigma X$ is the Stone-Čech compactification of $X$ if and only if $\sigma X$ is 
compact, or equivalently, $\sigma X$ is regular. Since $\sigma X$ is regular relative to $\sigma X \backslash X$ by Theorem 6.2 , then the following corollary is an easy consequence of Theorem 6.4.

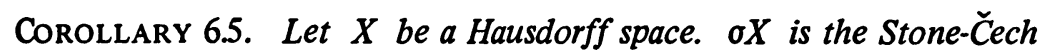
compactification of $X$ if and only if $X$ is regular and every free open ultrafilter is regular.

It is straightforward to verify that a Hausdorff space $X$ is regular and every free open ultrafilter is regular if and only if every closed nowhere dense subspace of $X$ is compact. Thus an interesting byproduct of the concept "regular relative to a subset" is a new proof of the following characterization due to Katětov [K2].

COROLlARY 6.6.(KATĚTOv). The Fomin extension of a Hausdorff space $X$ is the Stone-Čech compactification of $X$ if and only if every closed nowhere dense subspace of $X$ is compact.

Problem. Prove or disprove that an extension $Y$ of $X$ that is regular relative to $Y \backslash X$ is a strict extension.

The converse of the problem is false; in fact, there are semiregular Hausdorff (and hence, strict) extensions that are not regular relative to the remainder. Consider any noncompact, minimal Hausdorff space $Y$ with a dense subspace $X$ of isolated points (cf. Example 3.14 in [BPS]). By Corollary 6.5, $\sigma X$ is the Stone-Čech compactification of $X$ and hence, is not projectively larger than any noncompact space. So, $Y$ is not regular relative to $Y X$.

We now define two other regularity conditions, both of which were introduced by Votaw [V]. An extension $Y$ of $X$ has relatively regular remainder if for each $y \in Y$ and each open set $U$ containing $y$, there are open sets $V, W$ containing $y$ such that $V \subseteq W, Y \backslash X \subseteq W$, and $\operatorname{cl}_{W} V \subseteq U . Y$ has relatively completely regular remainder if for each $y \in Y$ and each open set $U$ containing $y$, there is an open set $W$ containing $y$ and a continuous function $f: W \rightarrow[0,1]$ such that $Y X \subseteq W, f(y)=0$ and $f(W U) \subseteq\{1\}$. It is not difficult to show that an extension with a relatively completely regular remainder has a relatively regular remainder. Moreover, since

$$
\mathrm{cl}_{W} V=W \cap \mathrm{cl}_{\boldsymbol{Y}} V \supseteq\left(\mathrm{cl}_{\boldsymbol{Y}} V\right) \backslash X \text { for } V \subseteq W,
$$

it is clear that an extension with relatively regular remainder is regular relative to $Y X$.

THEOREM 6.7. If $Y$ is an extension of $X$ with a relatively regular remainder, then $Y$ is a strict extension of $X$. 
Proof. Let $y \in U \subseteq Y$ where $U$ is open. There are open sets $V, W$ containing $y$ such that $V \subseteq W, Y X \subseteq W$, and $\operatorname{cl}_{W} V \subseteq U$. Then $y \in$ $o_{Y}(V \cap X) \subseteq \mathrm{cl}_{W} V \subseteq U$.

Votaw [V] has developed a generalized type of uniformity, called parauniformity, that generates all the $H$-closed extensions with relatively completely regular remainder.

We close this section with an interesting result concerning the subsets of the projectively ordered, complete upper semilattice of $H$-closed extensions of $X$ with relatively (completely) regular remainder (which are regular relative to the remainder).

THEOREM 6.8. Let A be a family of H-closed extensions of $X$. If each extension in $A$ is regular relative to its remainder (resp., has a relatively regular remainder, has a relatively completely regular remainder), then the projective supremum of $\mathrm{A}$, denoted by $\vee \mathrm{A}$, is an H-closed extension which is regular relative to its remainder (resp., has a relatively regular remainder, has a relatively completely regular remainder).

Proof. Parovičenko [P] showed that $V A$ is an $H$-closed extension of $X$ isomorphic to $\operatorname{cl}_{P}(e(X))$ where $P$ is the product space $\Pi\{Y: Y \in A\}$ and $e: X \rightarrow P$ is the embedding of $X$ defined by $e(x)(Y)=x$ for every $x \in X$ and every $Y \in A$. Let $Z$ denote the subspace $\operatorname{cl}_{P}(e(X))$ of $P$ with $e(x)$ and $x$ identified, for each $x \in X$.

Suppose that each $Y \in A$ is regular relative to $Y X$. Then the Katětov function $f_{Y}$ of $Y$ can be factored through $\sigma X$. By a well-known property of product spaces, there is a continuous function $g: \sigma X \rightarrow P$ such that $g(y)(Y)=$ $f_{Y}(y)$ for every $y \in \sigma X$ and every $Y \in A$. Since $g$ extends $e$, then $g$ is a continuous function from $\sigma X$ to $Z$ extending the identity function on $X$. Thus, $g$ is the Katětov function of $Z$, and by Theorem $6.2, Z$ is regular relative to $Z \backslash X$.

Now suppose that each $Y \in A$ has relatively completely regular remainder. Let $z \in Z$ and $U$ be an open set containing $z$. There is a finite subset $F \subseteq A$ and there are open neighborhoods $U_{Y}$ of $\pi_{Y}(z)$, for $Y \in F$, such that $z \in$ $\left.\left(\bigcap_{\left\{\pi_{Y}^{-1}\right.}\left(U_{Y}\right): Y \in F\right\}\right) \cap Z \subseteq U$, where $\pi_{Y}$ is the projection of $P$ onto $Y$. Then, for each $Y \in F$, there are open neighborhoods $W_{Y}$ of $\pi_{Y}(z)$ and a continuous function $g_{Y}: W_{Y} \rightarrow[0,1]$ such that $Y X \subseteq W_{Y}, g_{Y}\left(\pi_{Y}(z)\right)=0$, and $g_{Y}\left(W_{Y} \backslash U_{Y}\right) \subseteq\{1\}$. We now show that $Z l e(X) \subseteq \bigcap\left\{\pi_{Y}^{-1}\left(W_{Y}\right): Y \in F\right\}$. To do this it suffices to show that

$$
Z \backslash e(X) \subseteq \Pi\{Y \backslash X: Y \in A\},
$$

since $Y X \subseteq W_{Y}$ for $Y \in F$. Since $\pi_{Y} l e(X)$ is a homeomorphism from $e(X)$ 
onto $X$ for each $Y \in F$, then by $1.8, \pi_{Y}(Z l e(X)) \subseteq Y X$ implying

$$
Z \backslash e(X) \subseteq \bigcap\left\{\pi_{Y}^{-1}(Y X): Y \in A\right\}=\Pi\{Y \backslash X: Y \in A\} .
$$

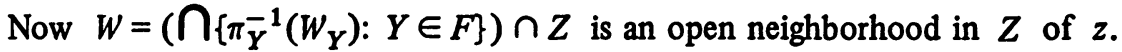
Let $g=\sup \left\{g_{Y} \circ \pi_{Y} \mid W: Y \in F\right\}$. Then $g: W \rightarrow[0,1]$ is a continuous function, $g(z)=0$, and $g(W \backslash U) \subseteq\{1\}$. Thus, $\vee A$ has relatively completely regular remainder. The proof for relatively regular remainder is similar.

7. Subextensions of the Fomin extension and the Banaschewski extension. Harris $[\mathrm{H}]$ has defined the concepts of $p$-cover (an open cover with the property that the union of some finite subcollection is dense), $p$-filter (a free open ultrafilter or an open neighborhood filter of a point), p-map (a continuous function with the property that the inverse image of a $p$-cover is a $p$-cover) and used them to prove some results for the Katětov extension which are analogous to corresponding results for the Stone-Čech compactification [GJ, Theorems 6.4 and 6.7]. These results by Harris are contained in the next theorem.

TheOREM 7.1 [H, TheOREM E]. Let $T$ be a simple Hausdorff extension of a space $X$. Consider the following conditions.

(a) Every p-map from $X$ into an H-closed space has an extension to $T$.

(b) $\kappa T$ and $k X$ are isomorphic as extensions of $X$.

(c) There is a homeomorphism from $T$ into $\mathrm{kX}$ that leaves $X$ pointwise fixed.

(d) Every p-cover of $X$ extends to an open cover of $T$.

(e) Every point of $T$ is the limit of a unique p-filter. Then (a), (b), (c), and (d) are equivalent and each implies (e).

In this section we characterize those Hausdorff (not necessarily simple) extensions of $X$ which satisfy either of the conditions (a), (d), or (e) of Theorem 7.1 and develop the relationships (b) and (c) for the Fomin extension and the Banaschewski extension. If $Y$ and $Z$ are extensions of $X$, we write $X \subseteq Y \subseteq Z$ to mean there is a homeomorphism from $Y$ into $Z$ that leaves $X$ pointwise fixed and $Y={ }_{X} Z$ if $Y$ and $Z$ are isomorphic as extensions of $X$. We now characterize those Hausdorff extensions of $X$ satisfying condition (a) of Theorem 7.1.

Corollary 7.2. Let $T$ be a Hausdorff extension of $X$. The following are equivalent:

(a) Every p-map from $X$ into an $H$-closed space has an extension to $T$.

(b) $X \subseteq T \subseteq \kappa X$.

(c) $k T={ }_{X} k X$. 
In the next theorem, we characterize those Hausdorff extensions satisfying condition (d) of Theorem 7.1.

THEOREM 7.3. Let $T$ be a Hausdorff extension of $X$. The following are equivalent:

(a) Every p-cover of $X$ extends to an open cover of $T$.

(b) $X \subseteq T \subseteq Y$ for some H-closed extension $Y$ of $X$ which is S-equivalent (see §3) to $\kappa X$.

(c) For each $y \in T, O^{y}$ is a p-filter on $X$.

(d) $\kappa T$ and $\kappa X$ are S-equivalent extensions of $X$.

Proof. The proof is omitted as it is similar to and simpler than the proof (which is included) of the forthcoming Theorem 7.4.

We now characterize those Hausdorff extensions satisfying condition (e) of Theorem 7.1.

THEOREM 7.4. Let $T$ be a Hausdorff extension of $X$. The following are equivalent:

(a) Every point of $T$ is the limit of a unique p-filter, i.e., for each $y \in T$, $O^{y}$ is contained in a unique p-filter.

(b) $X \subseteq T \subseteq Y$ for some H-closed extension $Y$ of $X$ which is $\theta$-isomorphic (see §3) to $\kappa X$.

(c) $\kappa T$ and $\kappa X$ are $\theta$-isomorphic extensions of $X$.

(d) For each $p$-cover $A$ of $X$, $\{\operatorname{int}(\mathrm{cl}(U)): U \in A\}$ can be extended to an open cover of $T$.

Proof. Since $X \subseteq T \subseteq \kappa T$, (c) implies (b) follows immediately.

(b) implies (d). By Theorem 3.5, the Katětov function $g: k X \rightarrow Y$ is a continuous bijection. By the definition of $g$, for $y \in Y \backslash X, g^{-1}(y)=$ $\left\{z \in \kappa X \backslash X: O^{y} \subseteq z\right\}$. By Lemma 1.9, for $y \in M X$, \{open $U$ : int $\left.(\operatorname{cl} U) \in O^{y}\right\}$ (denoted by $z_{y}$ ) is an open ultrafilter. If $A$ is a $p$-cover of $X$, then there is a subfamily $\left\{U_{1}, \cdots, U_{n}\right\}$ of $A$ whose union is dense in $X$. Thus, for $U=\bigcup_{i=1}^{n} U_{i}, k X \backslash X \subseteq O_{U}=\bigcup_{i=1}^{n} O_{U_{i}}$. So, for each $y \in Y \backslash$, there is $V \in$ A such that $V \in z_{y}$, implying int $(\operatorname{cl}(V)) \in O^{y}$. This shows that $\left\{O_{Y}(\operatorname{int}(\mathrm{cl}(U)))\right.$ : $U \in A\}$ is an open cover of $Y$.

(d) implies (a). Since $O^{x}=N_{x}$ for $x \in X$, then it remains to show that $O^{y}$ (relative to $T$ ), for $y \in T \backslash$, is contained in a unique open ultrafilter. Assume that, for some $y \in T \backslash X, O^{y}$ is not contained in a unique open ultrafilter. Then, by $1.9, z_{y}=$ \{open $\left.U: \operatorname{int}(\operatorname{cl}(U)) \in O^{y}\right\}$ is not an open ultrafilter. By [Wi, 12G], for some open set $U$ in $X, U \notin z_{y}$ and $\left.X\right|^{\prime} l_{X} U \notin z_{y}$. For each $u \in \operatorname{cl}_{X} U \backslash U$, there is an open set $V_{u}$ of $u$ such that $\operatorname{int}\left(\operatorname{cl}\left(V_{u}\right)\right) \notin O^{y}$. Now, $A=\left\{U, X \operatorname{lcl}_{X} U\right\} \cup\left\{V_{u}: u \in \mathrm{cl}_{X} U \cup U\right\}$ is a $p$-cover and $\{\operatorname{int}(\operatorname{cl}(V))$ : 
$V \in A$ A cannot be extended to an open cover of $T$.

(a) implies (c). $k T$ is an $H$-closed extension of $X$; so, let $f: \kappa X \rightarrow \kappa T$ be the Katětov function of $k T$. By Theorem 3.5, it suffices to show that $f$ is one-to-one. For $y \in T, O_{k T}^{y}=O_{T}^{y}$; so, $f^{-1}(y)$ is a singleton by assumption (a). Suppose $y \in \kappa T \backslash T$, then $\{U \cap T: U$ open in $k T$ and $y \in U\}$ is a free open ultrafilter on $T$ and since $X$ is dense in $T$, it easily follows that $O_{\kappa T}^{y}$ is a free open ultrafilter on $X$. Thus, $f^{-1}(y)$ is a singleton for $y \in \kappa T \backslash T$.

REMARK 7.5. It follows immediately from Corollary 7.2 and Theorems 7.3 and 7.4 that, for a Hausdorff extension $T$ of $X$,

(i) if every $p$-map from $X$ into an $H$-closed space can be extended to $T$, then every $p$-cover of $X$ extends to an open cover of $T$ and

(ii) if every $p$-cover of $X$ extends to an open cover of $T$, then every point of $T$ is the unique limit of a $p$-filter on $X$.

The converses of (i) and (ii) are false for arbitrary Hausdorff extensions of $X$. To show the converse of (i) is false, it suffices to show there is a Hausdorff space $X$ for which $k X$ and $\sigma X$ are not isomorphic, but this occurs when $X$ is Hausdorff but not locally $H$-closed (Corollary 4.3). The converse of (ii) is false even if $T$ is a strict (or simple) extension as demonstrated by a space $X$ in which the $\theta$-isomorphism class of $k X$ contains two nonisomorphic strict (and hence two nonisomorphic simple) extensions; such a space $X$ is described in Example 3.8.

Conditions (b) and (c) of Corollary 7.2 are stated in terms of the Katětov extension $\kappa X$, and an interesting inquiry is whether (b) and (c) of Corollary 7.2 are equivalent whenever $\kappa X$ is replaced by the Fomin extension $\sigma X$ or the Banaschewski-Fomin-Shanin extension $\mu X$. This inquiry is resolved by the next theorem and following example.

THEOREM 7.6. Let $T$ be a Hausdorff extension of $X$.

(a) If $\sigma T={ }_{X} \sigma X$, then $X \subseteq T \subseteq \sigma X$.

(b) Suppose $X$ is semiregular. Then $\mu T=_{X} \mu X$ if and only if $X \subseteq T \subseteq \mu X$.

Proof. The proof of (a) and the corresponding part of (b) is straightforward. Suppose $X$ is semiregular and $X \subseteq T \subseteq \mu X . T$ is semiregular since semiregular is hereditary on dense subspaces ([K1], $[\mathrm{St}])$. Since $(\kappa T)_{s}=\mu T$ and $(\kappa X)_{s}=\mu X$ and since $\kappa T$ is an $H$-closed extension of $X$, then by Theorem 3.5, $\mu X={ }_{X} \mu T$ if $k X$ and $\kappa T$ are $\theta$-isomorphic extensions of $X$. Let $f: \kappa X \rightarrow \kappa T$ be the Katětov function for $\kappa T$. Since $f \mid X$ is a homeomorphism, then by $1.8, f^{-1}(y)$ is a singleton for $y \in X$. For $y \in \kappa T \backslash X, f^{-1}(y)=$ $\left\{p \in \kappa X \backslash X: p \supseteq O^{y}\right.$ (relative to $\kappa T$ as an extension of $X$ )\}. If $y \in \kappa T \backslash T$, then $\{U \cap T: U$ open in $\kappa T$ and $y \in U\}$ is a free open ultrafilter on $T$, and as $X$ is dense in $T, O^{y}$ is a free open ultrafilter on $X$. This shows $f^{-1}(y)$ 
is a singleton for $y \in \kappa T \backslash T$. Since $X \subseteq T \subseteq \mu X$, the Katětov function for $\mu X$ (from $\kappa X$ ) is the identity function, and for $y \in T\left(X, O_{k T}^{y}=O_{T}^{y}=O_{\mu X}^{y}\right.$; then $f^{-1}(y)$ is a singleton for $y \in T \backslash X$. This shows that $f$ is one-to-one. By Theorem 3.5, $\kappa X$ and $k T$ are $\theta$-isomorphic extensions of $X$.

EXAMPLE 7.7. This is an example of a Hausdorff space $X$ that demonstrates the falsity of the converse of condition (a) of Theorem 7.6. Let $X$ be the positive integers with the discrete topology, and let $T=\sigma X \backslash\{y\}$ for some $y \in \sigma X \backslash X$. Since every nowhere dense closed subset of $X$ is compact, then by Corollary 6.6, $\sigma X$ is compact Hausdorff. But as noted by Flachsmeyer [F, p. 373], $\kappa T=\sigma T$ and $\kappa T$ is not compact, hence, $\sigma T \neq_{X} \sigma X$.

8. A lattice isomorphism and $\sigma X \backslash X$. We now turn our attention to the $\theta$-isomorphism classes of $H$-closed extensions and show that, for locally $H$-closed spaces $X$ and $Y$, the collections of such classes are lattices and are lattice isomorphic if and only if $\sigma X \backslash X$ and $\sigma Y \backslash Y$ are homeomorphic.

Let $Y$ be an $H$-closed extension of $X, f$ the Katětov function of $Y$, and $R_{Y}=\{(s, t): f(s)=f(t)\}$. Let $[Y]$ denote the $\theta$-isomorphism class containing $Y$ (see §3). If $Z$ is also an $H$-closed extension of $X$, define $[Y] \leqslant$ [Z] if and only if $R_{Z} \subseteq R_{Y}$. Clearly, the relation $\leqslant$ is a partial order on the collection of $\theta$-isomorphism classes of $H$-closed extensions of $X$; this collection is denoted by $\theta[X]$. In fact, more is true as indicated in the next theorem.

THEOREM 8.1. Let $X$ be a Hausdorff space.

(a) The relation $\leqslant$ is a partial order on $\theta[X]$ and every nonempty subset of $\theta[X]$ has a supremum in $\theta[X]$.

(b) If $X$ is locally $H$-closed, then $\theta[X]$ is a complete lattice relative to $\leqslant$.

Proof. The first part of statement (a) is noted in the paragraph preceding Theorem 8.1. To prove the second part of statement (a), let $\varnothing \neq \Gamma \subseteq \theta[X]$. Let $A$ be a set of representatives, one from each element of $\Gamma$. By the second remark of $\S 2$, the supremum, denoted as $Z$, of $A$ exists and $R_{Z}=$ $\left.\bigcap_{\left\{R_{Y}\right.}: Y \in A\right\}$. So, $[Z] \geqslant[Y]$ for every $Y \in A$. Also, if $W$ is an $H$-closed extension of $X$ and if $[W] \geqslant[Y]$ for every $Y \in A$, then $R_{W} \supseteq \bigcap\left\{R_{Y}: Y \in\right.$ $\mathrm{A}\}=R_{Z}$. So $[Z]$ is a supremum of $\Gamma$ relative to $\leqslant$. To prove (b), let $X$ be locally $H$-closed and let $\varnothing \neq \Gamma \subseteq \theta[X]$. There is a one-point $H$-closed extension of $X$ which is a lower bound for $\Gamma$. So, there is a greatest lower bound for $\Gamma$ in $\theta[X]$, by (a) above. Thus, $\theta[X]$ is a complete lattice.

Note that the partial order $\leqslant$ on $\theta[X]$ corresponds to $\theta$-projective order, for the obvious extension of the concept of projective order to $\theta$-projective order. Moreover, the order $\leqslant$ on $\theta[X]$ corresponds to projective order 
on the set consisting of a projective maximum from each class in $\theta[X]$ (the existence of such a maximum element was noted in a paragraph preceding Theorem 3.7).

Recall that a lattice isomorphism is a bijection that preserves finite supremums (if they exist) and finite infimums (if they exist). If $X$ is a locally compact Hausdorff space, it is well known that the collection of Hausdorff compactifications of $X$ forms a complete lattice relative to the projective order. For locally compact Hausdorff spaces $X$ and $Y$, Magill [M] has shown that the families of Hausdorff compactifications of $X$ and $Y$ are lattice isomorphic if and only if $\beta X \backslash X=\beta Y \backslash$, where $\beta X$ denotes the Stone-Čech compactification of $X$. This result will now be extended to $\theta[X], \theta[Y]$ and $\sigma X \backslash X, \sigma Y \backslash Y$, for locally $H$-closed spaces.

THEOREM 8.2. Let $X$ and $Y$ be locally H-closed spaces. Then $\theta[X]$ is lattice isomorphic to $\theta[Y]$ if and only if $\sigma X \backslash X=\sigma Y Y$.

Proof. Since $X$ and $Y$ are locally $H$-closed, then by Theorem 5.6, $\sigma X \backslash X$ and $\sigma Y \backslash Y$ are closed, compact subspaces of $\sigma X$ and $\sigma Y$, respectively. By Theorem 2.1 and 3.5, there is a bijection between $\theta[X]$ and the free, saturated equivalence relations $R$ on $k X$ such that $R(x)=\{x\}$ for $x \in X$. But there is a bijection between these types of equivalence relations on $X$ and partitions by free, saturated subsets of $\kappa X \backslash X$ (and, hence, of $\sigma X \backslash X)$. It follows by Theorem 5.4 that there is a bijection between $\theta[X]$ and the set of partitions of $\sigma X \backslash X$ by closed subsets. A similar statement holds for $Y, \theta[Y]$, and $\sigma Y Y$. Now a homeomorphism of $\sigma X \backslash X$ and $\sigma Y \backslash Y$ induces a lattice isomorphism of the sets of partitions of $\sigma X \backslash X$ and $\sigma Y \backslash Y$ by closed subsets, and it is clear that this lattice isomorphism extends to a lattice isomorphism of $\theta[X]$ and $\theta[Y]$.

Conversely, suppose there is a lattice isomorphism between $\theta[X]$ and $\theta[Y]$. Then, by considering the $H$-closed extensions obtained by identifying two points at a time, we obtain a bijection $f: \sigma X \backslash X \rightarrow \sigma Y \backslash$, just as Magill [M] does for $\beta X \backslash X$ and $\beta Y Y$. But then, since elements of $\theta[X]$ correspond to partitions of $\sigma X \backslash X$ by closed sets, we can show that $f$ and $f^{-1}$ are closed, by using essentially the same argument Magill uses. Thus, $\sigma X \backslash X$ is homeomorphic to $\sigma Y Y$.

By Theorem 5.6, $p X$ is locally compact if and only if $X$ is locally $H$-closed, and by 5.10 and 5.11, $\sigma X \backslash X$ is homeomorphic to $\beta(p X) \backslash p X$ and to $\sigma(p X) \backslash p X$. Thus, we have a lattice isomorphism of $\theta[X]$ and $\theta[p X]$, for locally $H$-closed $X$. This leads to the question of whether the lattice isomorphism of $\theta[X]$ with $\theta[Y]$ is an extension of the lattice isomorphism of $K[p X]$ with $K[p Y]$, for locally $H$-closed $X$ and $Y$ with $\sigma X \backslash X$ 
homeomorphic to $\sigma Y Y$, where $K[p X]$ denotes the family of Hausdorff compactifications of $p X$. But it is not difficult to see that the homeomorphism of $\sigma X \backslash X$ with $\sigma Y \backslash Y$ uniquely determines both lattice isomorphisms through the "natural" identification of $\sigma X \backslash X$ with $\sigma(p X) \backslash p X$ and $\sigma Y \backslash Y$ with $\sigma(p Y) \backslash p Y$. (Consider the $H$-closed extensions of $X, Y$ and the compactifications of $p X$, $p Y$ obtained by identifying two points in $\sigma X \backslash X$ and the corresponding points in $\sigma Y \backslash Y$.) Thus, if a partition of $\sigma X \backslash X$ by closed subsets induces a compactification of $p X$, the corresponding partition of $\sigma Y \backslash Y$ induces a compactification of $p Y$. This, of course, implies that the answer to the question posed above is affirmative. We record this result, along with some others, in the following corollary.

COROLlaRY 8.3. Let $X$ and $Y$ be locally H-closed. The following are equivalent:

(a) $\sigma X \backslash X=\sigma Y \backslash Y$.

(b) $\beta(p X) \backslash p X=\beta(p Y) \backslash p Y$.

(c) $\theta[X]$ is lattice isomorphic to $\theta[Y]$.

(d) $\theta[p X]$ is lattice isomorphic to $\theta[p Y]$.

(e) $K[p X]$ is lattice isomorphic to $K[p Y]$.

(f) There is a lattice isomorphism $r: \theta[p X] \rightarrow \theta[p Y]$ such that $r(K[p X])$ $=K[p Y]$.

A fact mentioned in the paragraph preceding Corollary 8.3 is true without local $H$-closure in the hypothesis and is presented in the next proposition, the proof of which follows immediately from Lemma 5.3.

Proposition 8.4. If $X$ is a Hausdorff space, then $\theta[X]$ and $\theta[p X]$ are lattice isomorphic.

\section{REFERENCES}

B B. Banaschewski, Über Hausdorffsch-minimale Erweiterungen von Raümen, Arch. Math. 12 (1961), 355-365. MR 25 \#5490.

BPS M. P. Berri, J. R. Porter and R. M. Stephenson, Jr., A survey of minimal topological spaces, General Topology and its Relations to Modern Analysis and Algebra. III (Proc. Conf., Kanpur, 1968), Academia, Prague, 1971, pp. 93-114. MR 43 \#3985.

F1 J. Flachsmeyer, Zur Theorie der H-abgeschlossen Erweiterungen, Math. Z. 94 (1966), 349-381. MR 35 \#299.

F2 - Über Erweiterungen mit nulldimensional gelegenem Adjunkt, Contributions to Extension Theory of Topological Structures (Proc. Sympos., Berlin, 1967), Deutsch. Verlag Wissenschaften, Berlin, 1969, pp. 63-72. MR 40 \#2002.

Fo S. V. Fomin, Extensions of topological spaces, Ann. of Math. (2) 44 (1943), 471-480. MR 5, 45.

GJ L. Gillman and M. Jerison, Rings of continuous functions, University Ser. in Higher Math., Van Nostrand, Princeton, N. J., 1960. MR 22 \#6994. 
H D. Harris, Katětov extension as a functor, Math. Ann. 193 (1971), 171-175. MR 45 \#7678.

IF S. Iliadis and S. Fomin, The method of centered systems in the theory of topological spaces, Uspehi Mat. Nauk 21 (1966), no. 4 (130), 47-76= Russian Math. Surveys 21 (1966), no. 4, 37-62. MR 34 \#3526.

K1 M. Katětov, A note on semiregular and nearly regular spaces, Časopis Pěst. Mat. Fys. 72 (1947), 97-99. MR 9, 521.

$\mathrm{K} 2$ - On the equivalence of certain types of extensions of topological spaces, Časopis Pěst. Mat. Fys. 72 (1947), 101-106. MR 9, 522.

L C.-T. Liu, Absolutely closed spaces, Trans. Amer. Math. Soc. 130 (1968), 86-104. MR 36 \#2107.

M K. D. Magill, Jr., The lattice of compactifications of a locally compact space, Proc. London Math. Soc. (3) 18 (1968), 231-244. MR 37 \#483.

O F. Obreanu, Espaces localement absolument fermés, An. Acad. Repub. Pop. Române. Sect. Mat. Fiz. Chim. Ser. A 3 (1950), 375-394. (Romanian) MR 13, 484.

P I. I. Parovičenko, On suprema of families of H-closed extensions of Hausdorff spaces, Dokl. Akad. Nauk SSSR 193 (1970), 1241-1244 = Soviet Math. Dokl. 11 (1970), 1114-1118. MR 42 \#3752.

Po J. Porter, On locally H-closed spaces, Proc. London Math. Soc. (3) 20 (1970), 193-204. MR 41 \#1010.

PT J. Porter and J. D. Thomas, On H.closed and minimal Hausdorff spaces, Trans. Amer. Math. Soc. 138 (1969), 159-170. MR 38 \#6544.

PV J. Porter and C. Votaw, H.closed extensions. I, General Topology and Appl. 3 (1973), 211-224.

S1 N. A. Šanin, On special extensions of topological spaces, C. R. (Dokl.) Acad. Sci. URSS 38 (1943), 6-9. MR 5, 45.

S2 - On separation in topological spaces, C. R. (Dokl.) Acad. Sci. URSS 38 (1943), 110-113. MR 5, 46.

St M. H. Stone, Applications of the theory of Boolean rings to general topology, Trans. Amer. Math. Soc. 41 (1937), 374-481.

V C. Votaw, H-closed extensions as para-uniform completions, Ph.D. Dissertation, University of Kansas, Lawrence, Kan., 1971.

W A. Wilansky, Topology for analysis, Ginn, Waltham, Mass., 1970.

Wi S. Willard, General topology, Addison-Wesley, Reading, Mass., 1970. MR 41 \#9173.

DEPARTMENT OF MATHEMATICS, UNIVERSITY OF KANSAS, LAWRENCE, KANSAS 66044 (Current address of J. R. Porter)

Current address (Charles Votaw): Department of Mathematics, Fort Hays Kansas State College, Hays, Kansas 67601 